\title{
Scenario Decomposition Based Analysis of Next Generation Mobile Services
}

\author{
Dongchun Shin ${ }^{1}$, Jinbae $\mathrm{Kim}^{2}$, Seungwan $\mathrm{Ryu}^{1, \star}$, Donsung $\mathrm{Oh}^{3}$, \\ Joowan Lee ${ }^{1}$, and Minhyung Kang ${ }^{1}$ \\ ${ }^{1}$ Department of Information Systems, Chung-Ang University, Korea \\ ${ }^{2}$ Department of Computer\&Information, Anyang Technical College, Anyang, Korea \\ ${ }^{3}$ Mobile Telecommunications Research Division, ETRI, Taejon, Korea \\ \{dcshin, rush2384, expertz\}@cau.ac.kr, jbkim@ianyang.ac.kr, \\ dsoh@etri.re.kr
}

\begin{abstract}
The next-generation mobile services have not been studied actively yet because there is no clear definition what the next generation mobile communication and its services will be. In this paper, we propose a service analysis framework for eliciting key service functionalities and core technologies using given various future mobile service scenarios. The proposed analysis framework, called scenario based service analysis process (2SAP), consists of 4 sequential analysis steps, a scenario transformation, a scenario decomposition, an analysis of functions for each situation, and an elicitation of service functionalities.
\end{abstract}

\section{Introduction}

It is expected that the next generation mobile communication system will be a service-driven developed system differs from the technology-driven development approach of the second and the third generation mobile communication systems 1, 2. Therefore, in the service-driven system development process for next generation system, various possible future mobile communication service scenarios are generated first. Then core technologies and network elements required to realize those service scenarios are elicited through scenario based analysis. Therefore, we believe that it is imperative to have a systematic scenario analysis method in such service-driven system development approach.

In this paper, we propose a service analysis framework for eliciting key service functionalities and core technologies when various future mobile service scenarios are given. The proposed analysis framework, called scenario-based service analysis process (2SAP), consists of 4 sequential analysis steps, a scenario refinement, a scenario decomposition, an analysis of function for each situation, and an elicitation of service functionalities. Then the refined scenarios are divided into several sets of situations based on several criteria. Each situation is also divided into several scenes to elicit service functionalities that are required to realize such scenes. Finally, various basic core technologies that are required to implement each service functionality are obtained.

\footnotetext{
^ Prof. S. Ryu is the corresponding author.
} 
This paper is organized as follows. In next section, concept of scenario is introduced followed by generic process of scenario based system design approaches. In section 3, we propose the scenario based service analysis process (2SAP) for next generation mobile services. Finally, we conclude this paper with giving further study issues.

\section{Scenarios and Scenario Based System Design}

\section{$2.1 \quad$ Scenarios}

In designing systems we need better means to describe contexts of user activities. A possible approach is explicit envisioning and documentation of typical and significant user activities early and continuingly in the development process. Such descriptions are often called scenarios that can support reasoning about situations of use, even before those situations are actually created [3. A scenario is defined as a set of situations having common characteristics generated while using a system. In short, scenarios are stories about people and their activities. A scenario should contain basic characteristics such as agent (or users), proper objectives to use, and operational conditions [4. In other words, scenarios could highlight goals, required procedures and role of users and subsystems by describing behavior of the system and activities of users. Such behaviors and activities include what users try to do with the system, what procedures are required or not adopted, and to be carried out to achieve, and what interpretations users make of what happens to them.

\subsection{Scenario Based System Design}

The basic idea behind scenario-based methods is that descriptions of technologies in use are essential in discussing and analyzing how those technologies could be used for reshaping of their activities. A scenario is used to analyze system requirement because of following properties [3].

- Scenarios are concrete but rough. On the one hand, a scenario could be a concrete design proposal that can be evaluated and improved by the designer. On the other hand, it is rough enough for designers to change it easily and to design details of the system later.

- Scenarios maintain an orientation to users and their needs. Since scenarios are work-oriented design objects that describes systems in terms of the work that users will try to do when they use those systems, it makes the design work remain with the needs and concerns of users. Therefore, scenarios can be made even more effective as work-oriented design objects when users are directly involved in creating them.

- A narrative scenario is written to evoke an image of people doing things, pursuing goals, using technology in support of these goals. The story enables readers to empathize with the people in the situation, which in turn leads to questions about motivations, intentions, reactions, and satisfaction. 
The concrete and work-oriented nature of scenarios gives effective representation means for human-centered design activities, particularly when these activities include participation of end-users. Because of the fore-mentioned properties of scenarios, we can get various benefits described below from the scenario-based requirement analysis in designing a system.

- It gives useful information by decomposing complex problems into small sub-problems systematically. In addition, since useful information can be obtained from various problems independently, it is possible to synthesize requirements from technologies extracted from scenarios.

- By analyzing coherency of scenarios, it is possible to discover distinct objects and operation conditions that may give effective ways to avoid conflict among end users.

- It provides basic verification tools via coherency analysis and completeness of well-defined scenarios.

- It provides effective and rapid communication ways about system usage and other concerning matters between all person involved in system development work.

\subsection{A generic Scenario Based Design Approach}

The scenario-based design is a design approach that describes future system usage at the beginning of system development process. Similar to user-centric design approaches, the scenario based design approach focuses on how people use the target system to perform their works and activities not on detailed functionalities. The scenario based design approach consists of three sequential steps, the analysis step, the design step and the prototyping and evaluation step.

- In the analysis step, general understanding of the current problem status is acquired from the problem scenario and related claims. The problem scenario describes participants (called actors) involved in meaningful activities, and the related claims and characteristics of the current status that may result significant effects to actors. The problem scenario is transformed and refined repeatedly by means of repetitive design attempts. Then a root concept that supports detailed analysis and refinement of the claims by describing essential aspects of initial vision is derived from the initial requirement analysis. Several important elements that constitute the root concept are a high-level vision, basic rationale, and stakeholder group.

- The design step is consists of three activity design stages, the activity design stage, the information design stage and the reciprocal action design stage.

- The activity design stage describes how the activities are improved or how does it changed by applying usable technologies. In this stage, new activities are generated by analyzing current meaningful activities. Then, activity scenario and related claims are obtained in this stage. 
- Information design stage describes how work information is organized and/or created while users are using the system. To achieve this purpose, special treatment of beginners or some who has no experience to use the system is required. In this stage, information scenarios and related claims are obtained.

- The last stage of design step is the reciprocal action design stage. This stage will take concrete shape when detailed exchanged items between users and the system are specified and listed.

- Usage list is generated from scenarios and claims. Usage list can be divided into several detailed operations corresponding to representative operation situation. Then target usage objective is assigned to each detailed operation. Design scenarios provide realistic work situations, whereas claims make it possible to analyze those scenarios in terms of expected usage objectives. When a prototype has sufficient functionalities, detailed operations are evaluated and compared with target objectives.

Figure 1 shows a generic scenario-based design framework.

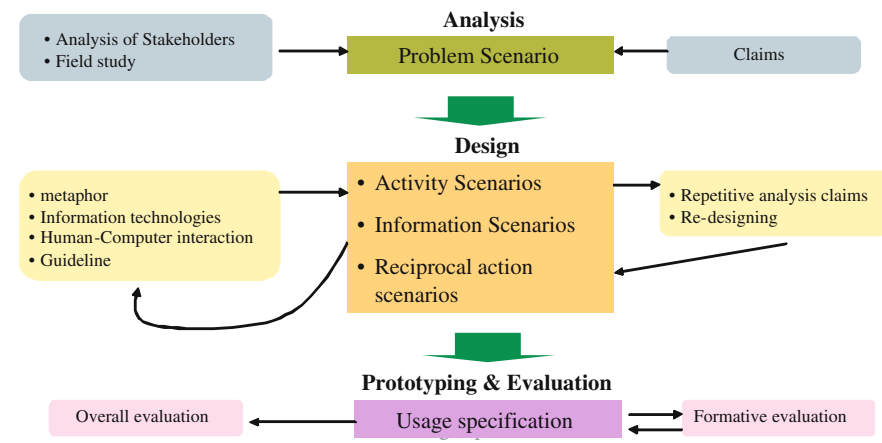

Fig. 1. A generic scenario-based design framework

\section{Scenario Based Service Analysis Process}

In this section we propose the scenario based analysis method for next generation mobile services, called the scenario-based service analysis process (2SAP). The proposed analysis method consists of four sequential steps, the scenario refinement process, the scenario decomposition process, the functional analysis process for each situation, and the service functionalities elicitation process. In the first process, various crude narrative scenarios are refined or better analysis. In the second process, based on several criteria, each scenario is decompose into a set of situations having common characteristics generated while using a system. In the third process, each situation obtained in previous process is also divided into several scenes to elicit service functionalities that are required to realize such scenes. Finally, various basic core technologies that are required to implement each service functionality are obtained in the last process. 
In this paper, initial and refined scenarios are described in natural language, and then expressed by scenario trees in the analysis step. Finally it is modelled as a use case diagram in both of functionality analysis and core technologies and service functionalities elicitation processes.

\subsection{Scenario Refinement}

In order to obtain core technologies and service functionalities of next generation mobile communication systems, various crude next generation mobile service scenarios are generated. These scenarios are written as a narrative story with lots of modifiers such as adjectives and adverbs. Although the "story" type crude service scenario is easy to understand, it is hard to get core technologies and service functionalities via analyzing such service scenarios. Thus in the scenario refinement process, initially generated story type scenario is refined to a concise one containing essential and necessary contents. In particular, all modifiers exists in a scenario are removed because they are used to make the scenario elegant and understandable for people. Then, the refined scenario is transformed into an enumerative type containing list of different situations. A possible initial story type service scenario and a refined and transformed enumerative scenario are shown in figure 2 and 3 respectively.

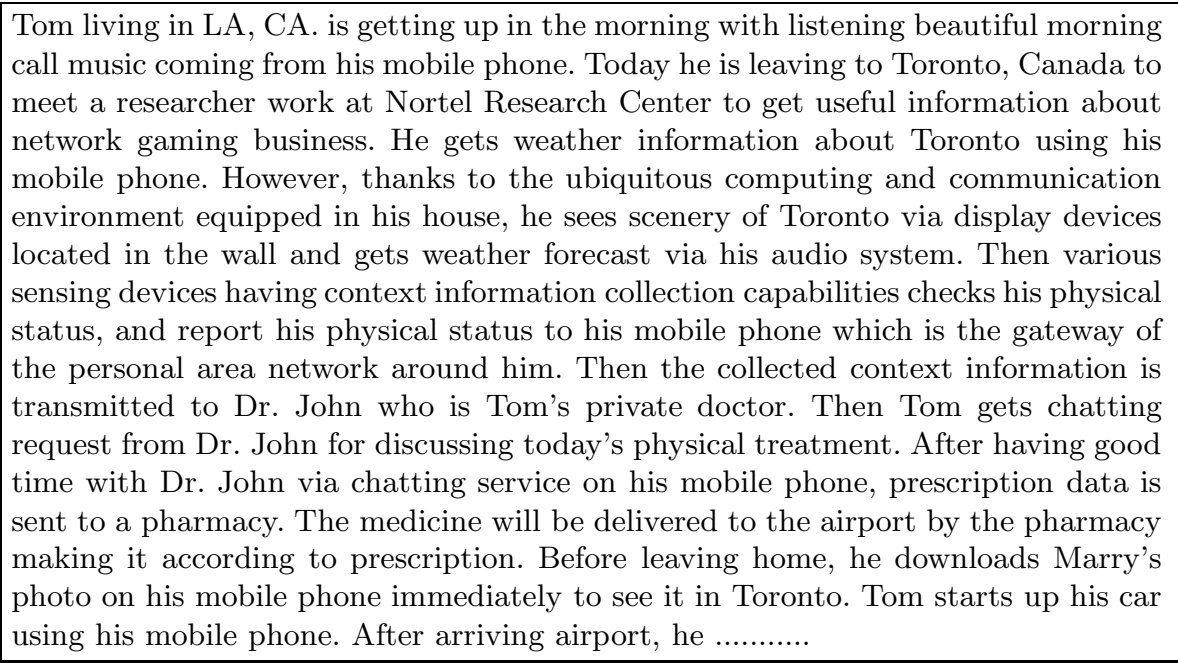

Fig. 2. An example narrative story type scenario

\subsection{Scenario Decomposition}

In previous process a scenario is refined and transformed into an enumerative type having list of different situations. Although it is possible to analyze all the listed situations together, a better and efficient approach is to analyze it after decomposing the whole scenario into a number of small sub-problems. Therefore, 
1. Tome get alarming service from his mobile phone.

2. Tom gets weather information about place which he is planning to visit.

3. Physical status of him is sent to his private doctor, and discuss with him via chatting service. Prescription is sent to pharmacy and medicine will be delivered to the airport.

4. Download photos of his daughter on his mobile phone.

5. .....

Fig. 3. An example of refined and transformed scenario into an enumerative one

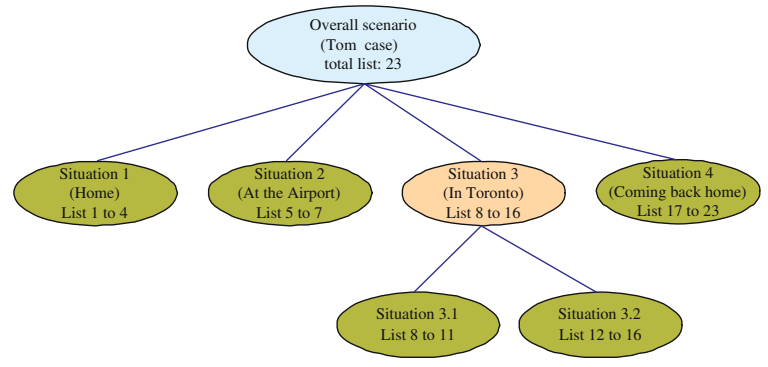

Fig. 4. An example of scenario tree representation based on decomposition and grouping approach

in 2SAP methods, the refined scenario is decomposed into a number of groups of situations based on coherency of them using grouping criteria.

The fundamental grouping criteria are location of a user, devices, and time. For example, in most cases, a new service is initiated when location and/or devices are changed. In addition, when, time especially date, is changed, it is highly probable to begin a new situation. However, this grouping task should be conducted carefully by taking circumstances of such changes on location, time and devices. When there are groups having larger numbers of list than other groups, they are decomposed into small sub-groups by applying the secondary decomposition criteria such as the number of actors and objectives of each situation. Figure 4 shows an example of scenario decomposition and grouping process.

\subsection{Functional Analysis of Each Situation}

In order to get service functionalities from situations described at the end nodes of the scenario tree, it is required to decompose each situation into smaller units called scene. A scene is defined as a target service moment that we are interested in. A scene is thus corresponds service functionality that is essential basis service technology felt directly by users [2]. The decomposition criteria used for getting several scenes from a situation are the initiation and/or releasing moment of services for a user gets it through his(or her) five senses. By means of intensive analysis of these scenes, we can obtain functionalities that make these scenes possible. We first establish a pool of possible service functionalities required to 
realize next generation mobile services using our previous research result [2]. Detailed functional analysis procedures for each situation are described below.

- Establish a pool of service functionalities. In this case, the pool can be established by assistant of expert groups or investigation of related technologies.

- derives scenes from a situation, and obtains service functionalities required to realize each scene using the pool of service functionalities.

- Gets a list of required functionalities for each scene.

- Finally, represents each scene (or a situation) as a use case diagram which describes relations between a user and service functions.

Table 1 gives example lists of scenes and required functionalities for the situation 1. Figure 5 also shows an example use case diagram of the situation 1.

Table 1. Example lists of scenes and required functionalities for the situation 1

\begin{tabular}{|l|l|l|}
\hline Scenes & Description & Functionalities \\
\hline Scene 1 & Gets alarming service via mobile phone & $\bullet$ Mobile phone alarming \\
\hline Scene 2 & Checking weather of the place to visit & $\bullet$ web browsing \\
\hline Scene 3 & $\begin{array}{l}\text { Health check \& data transferring to } \\
\text { a doctor }\end{array}$ & $\begin{array}{l}\bullet \text { Remote Control } \\
\bullet \text { data messaging \& file transfer }\end{array}$ \\
\hline Scene 4 & Chatting request from a doctor & $\bullet$ Chatting / Messenger \\
\hline Scene 5 & prescription is delivered to a pharmacist & $\bullet$ File transfer \\
\hline Scene 5 & Download daughter's photos & $\bullet$ File transfer \\
\hline
\end{tabular}

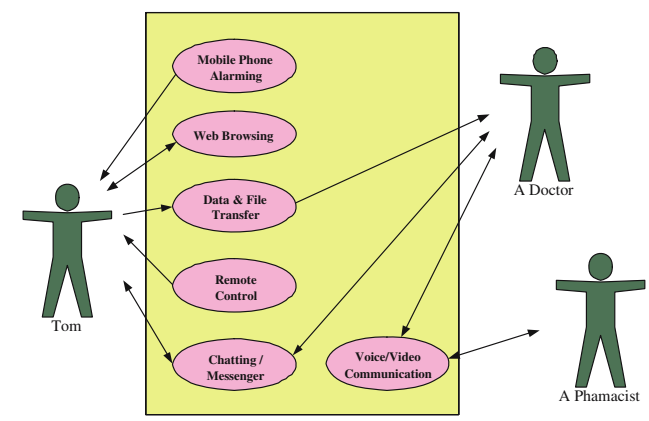

Fig. 5. An example use case diagram of the situation 1

\subsection{Elicitation of Service Technologies}

Several types of core technologies such as platform technologies, mobile terminal technologies, and service application technologies are necessary to implement service functionalities. Service technologies elicitation procedure is described as follow 
- Establish a pool of service technologies with assistant of expert groups or investigation of related reference books or technical documents.

- Derives service technologies for each service functionalities using use case diagrams obtained in previous process. If there is a service technology that is required in realizing a service functionality but does not exist in the pool, creates a new service technology and makes a detailed technological list.

- Gets a list of required service technologies for each service functionalities.

- Finally, for each service functionality, draw a use case diagram describes relations between a user and service technologies.

\section{Conclusion and Further Study Issues}

In this article, we propose a service analysis framework for eliciting key service functionalities and core technologies when various future mobile service scenarios are given. The proposed analysis framework, called scenario-based service analysis process (2SAP), consists of 4 sequential analysis steps, a scenario refinement, a scenario decomposition, an analysis of function for each situation, and an elicitation of service functionalities. We believe that our research activities and results on next generation mobile services, especially the 2SAP framework, will play important role in developing core mobile communication technologies as well as next generation ubiquitous mobile applications and services.

\section{References}

1. S. Arbanowski et al., "I-centric Communications: Personalization, Ambient Awareness, and Adaptability for Future Mobile Services," IEEE Comm. Magazine, 42(10), pp. 63-69, September, 2004.

2. S. Ryu et al., "Research Activities on the Next Generation Mobile Communications and Services in Korea," IEEE Comm. Magazine, 43(9), pp. 122-131, 2005.

3. M. Rosson and J. Caroll, The Human-Computer Interaction Handbook, Lawrence Erlbaum Associates, 2002.

4. H. Zhu and L. Jin, "Scenario Analysis in an Automated Tool for Requirements Engineering," Requirement Engineering, 5, pp. 2-22, 2000. 